\title{
FTIR and DSC studies of mechanically deformed $\beta$-PVDF films
}

\section{Authors: S. Lanceros-Mendez, J. F. Mano, A. M. Costa, and V. Hugo Schmidt}

This is an Accepted Manuscript of an article published in Journal of Macromolecular Science, Part B: Physics in 2001, available online: https://www.tandfonline.com/10.1081/MB-100106174.

S. Lanceros-Mendez, J. F. Mano, A. M. Costa, and V. Hugo Schmidt, "FTIR and DSC studies of mechanically deformed $\beta$-PVDF films," Journal of Macromolecular Science, Part B: Physics 40, 517-527 (2001). doi: 10.1081/MB-100106174.

Made available through Montana State University's ScholarWorks

scholarworks.montana.edu 


\title{
FTIR AND DSC STUDIES OF MECHANICALLY DEFORMED $\beta$-PVDF FILMS
}

\author{
S. Lanceros-Méndez, ${ }^{1}$ J. F. Mano, ${ }^{2, *}$ A. M. Costa, ${ }^{2}$ \\ and V. H. Schmidt ${ }^{3}$ \\ ${ }^{1}$ Physics Department, Minho University, 4710 Braga, Portugal \\ ${ }^{2}$ Polymer Engineering Department, Minho University, \\ 4800-058 Guimarães, Portugal \\ ${ }^{3}$ Physics Department, Montana State University, Bozeman, MT 59717
}

\begin{abstract}
Films of semicrystalline poly(vinylidene fluoride) (PVDF) in the $\beta$ phase were studied by Fourier transform infrared (FTIR) spectroscopy and differential scanning calorimetry (DSC). The main goal of this study was to improve the understanding of the structural changes that occur in $\beta$-PVDF during a mechanical deformation process. FTIR spectroscopy was used to examine the structural variations as a function of strain. DSC data allowed measurement of the melting temperatures and enthalpies of the material before and after deformation, providing information about the changes in the crystalline fraction. After the molecular vibrations were assigned to the corresponding vibrational modes, we investigated the energy and intensity variations of these vibrations at different deformations. A reorientation of the chains from perpendicular to parallel to the stress direction was observed to occur in the plastic region. During the deformation, a decrease in the degree of crystallinity of the material was observed, but the thickness of the lamellae did not change significantly.
\end{abstract}

Key Words: Mechanical anisotropy; Plastic deformation; PVDF; Vibrational modes. 


\section{INTRODUCTION}

Poly(vinylidene fluoride) (PVDF) has been intensely investigated because of its interesting ferroelectric properties and technological applications. PVDF can exist in several crystalline phases [1]. Of the five known modifications, the $\alpha$ - and $\beta$-phases are most common. The $\alpha$-form, the more common and stable, arises usually from the melt when PVDF crystallizes in quiescent conditions. The $\beta$-form offers the highest piezo-, pyro-, and ferroelectric properties. To obtain $\beta$ PVDF, a technological process involving stretching and poling of extruded thin sheets of the polymer is used, resulting in permanent polarization. Uniaxial stretching provides the alignment of molecular chains. An applied electric field up to $100 \mathrm{kV} / \mathrm{mm}$, at temperatures around $100^{\circ} \mathrm{C}$, causes the net polarization of PVDF, which is maintained once the material is cooled to room temperature [2].

Generally, this transformation does not give rise to samples composed completely of one polymorphic modification, but small amounts of the other phases are always present. The infrared spectra allow the various forms to be clearly distinguished by identifying some characteristic bands with the corresponding phases.

Deformation processes are intimately associated with the production of these kinds of films, which have applications as sensors and actuators. Such oriented films are often subjected to further stresses during service, and morphological changes may occur mainly in the direction perpendicular to the preferred orientation of the chains, in which the mechanical properties are weaker.

In this study, Fourier transform infrared (FTIR) experiments were carried out on $\beta$-PVDF films with different deformations induced at room temperature. Such information may be relevant in identifying changes in the anisotropy, degree of crystallinity, crystalline phase, and other morphological changes that occur during deformation. Complementary information was also obtained by performing differential scanning calorimetry (DSC) studies of the same samples.

The correlation between different properties (e.g., mechanical) and the microstructure of polymeric systems has been studied for many years. The present work constitutes a preliminary study within a broader project that intends to follow and understand the changes at the microstructural level of relevant plastic materials (with originally different morphologies) during deformation using a series of complementary techniques, including FTIR, DSC, atomic force microscopy, X-ray spectroscopy, and dielectric and mechanical relaxation spectroscopies.

\section{EXPERIMENTAL PROCEDURE}

The starting material was a $28-\mu \mathrm{m}$ thick commercial PVDF film from Measurements Specialties, Incorporated (Fairfield, NJ). The material was deformed to different degrees in the direction perpendicular to the initial chain orientation. 
Deformed samples were prepared using a T30K JJ instruments (Looyd Instruments, Southampton, UK) tensile testing machine. The 13-mm wide PVDF films were fixed (distance between clamps $40 \mathrm{~mm}$ ) and pulled apart at a constant speed of $2 \mathrm{~mm} / \mathrm{min}$. This strain rate is small enough to warrant negligible temperature elevation. Samples with different deformations in the range from $35 \%$ to $140 \%$ (break point) were prepared and studied.

Infrared spectra were obtained from 400 to $6000 \mathrm{~cm}^{-1}$ using polarized light in perpendicular $\perp$ and parallel $\|$ modes. In the parallel mode, the electric vector of the light is parallel to the prior chain orientation (and perpendicular to the draw direction); in the perpendicular mode, the electric vector of the light is perpendicular to the initial chain orientation. FTIR analysis was performed in transmission at room temperature on a Bruker $66 \mathrm{~V}$ spectrometer (Ettlinger, Germany), with a resolution of $1 \mathrm{~cm}^{-1}$.

Thermal analysis was carried out with a Setaram DSC 131 differential scanning calorimeter (Caluire, France) at a heating rate of $10^{\circ} \mathrm{C} / \mathrm{min}$ under nitrogen atmosphere. Samples were heated from room temperature to $250^{\circ} \mathrm{C}$. Melting temperatures and enthalpies were determined at the onset of the peaks and from the peak areas, respectively.

\section{RESULTS AND DISCUSSION}

The anisotropy of the original $\beta$-PVDF films is observed in the stress-strain mechanical experiments carried out in the two main directions (Fig. 1). In the longitudinal direction, the film shows a typical brittle behavior with a higher

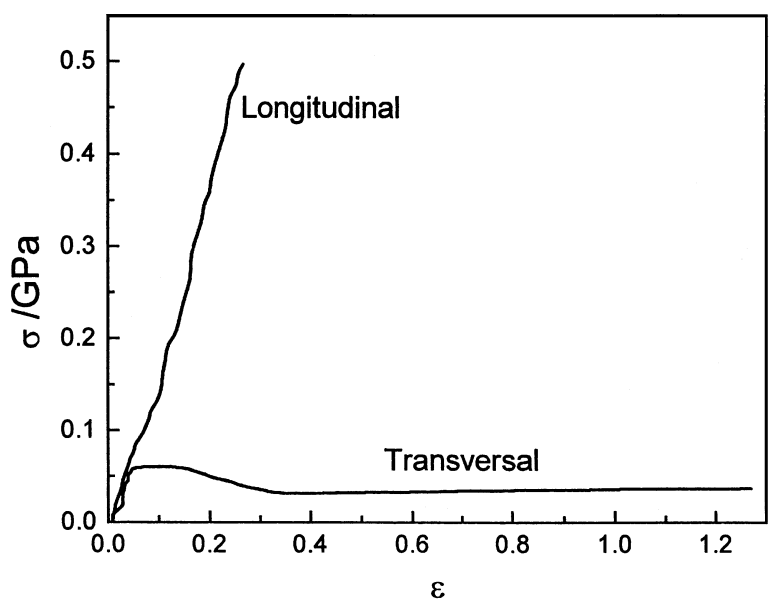

Figure 1. Stress-strain curves for $\beta$-PVDF in the two main directions obtained at $2 \mathrm{~mm} / \mathrm{min}$. Longitudinal means that the stress has the same direction as the original preferred chain orientation, and transversal means that the deformation is perpendicular to the preferred chain direction. 
ultimate stress and a lower strain at break than for the transversal direction. Therefore, the material enables higher deformations in the transversal direction.

The anisotropic behavior of the $\beta$-PVDF films was also analyzed by FTIR. First, the spectra of the virgin sample were analyzed in both polarization directions to assign some modes or spectral regions to specific molecular vibrations. Second, the effects on these molecular modes and on the different crystalline phases produced by the application of a well-defined strain were analyzed. These results were further compared with the DSC data, which gives complementary information on the effect of the strain on the modification within the crystalline fraction of the polymer.

\section{Identification of Vibrational Modes and Spectral Regions}

\section{0-1500- $\mathrm{cm}^{-1}$ Region}

In Fig. 2, the FTIR transmission spectra of virgin PVDF with the electric field vector both parallel and perpendicular to the chain direction are shown in the $400-1500-\mathrm{cm}^{-1}$ region. The observed pattern originates from oscillations of large parts of the skeleton and/or the skeleton and attached functional groups. Below $1500 \mathrm{~cm}^{-1}$, most single bonds absorb at similar frequencies, and the vibrations couple. Nevertheless, some modes and spectral regions have been identified

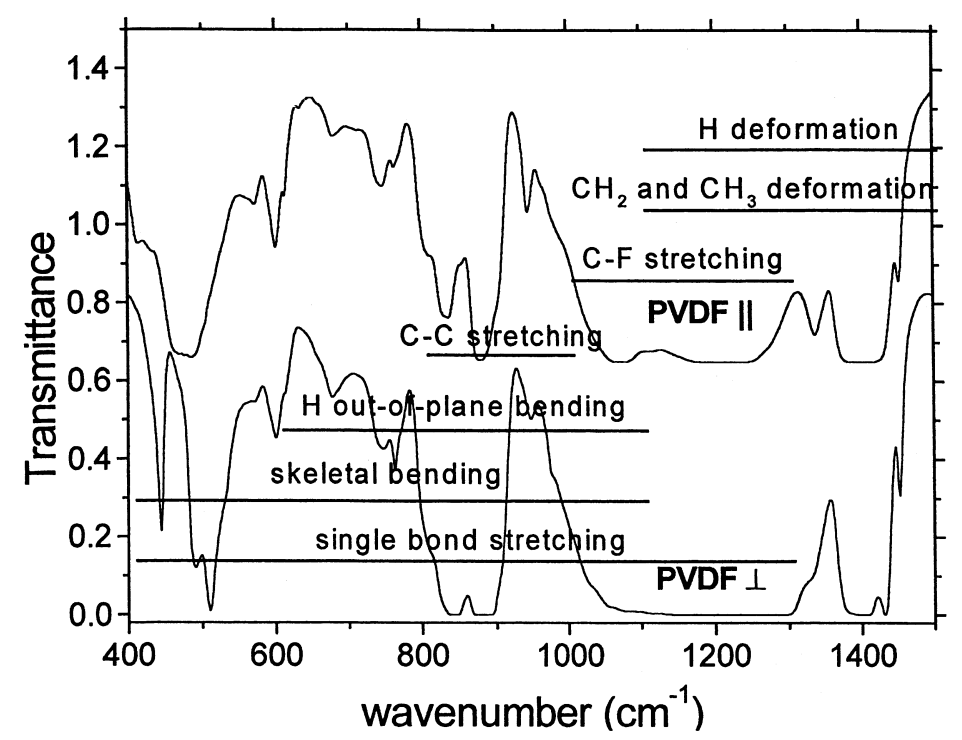

Figure 2. FTIR spectra of the original PVDF with polarized light. In the PVDF $\perp$ mode, the electric vector of the light is perpendicular to the chain orientation; in PVDF $\|$, the electric vector of the light is parallel to the chain orientation. Some typical vibration regions are also shown. 
with the help of the literature [3-9]. The results are summarized in Table 1 and Fig. 2, respectively.

The identified regions are rich in information on the conformational isomerism of the chain, providing information on the $\alpha$ - and $\beta$-phase content. Though the virgin sample was prepared to obtain the $\beta$-phase, some characteristic bands of the crystalline $\alpha$-phase have been observed at 763,615 , and 490 $\mathrm{cm}^{-1}$. The absorption band at $763 \mathrm{~cm}^{-1}$ is related to a rocking vibration [5]. The band at $615 \mathrm{~cm}^{-1}$ is assigned to a mixed mode of $\mathrm{CF}_{2}$ bending and $\mathrm{CCC}$ skeletal vibration; this peak is parallel to the chain axis [5,7]. The $490-\mathrm{cm}^{-1}$ band is related to bending and wagging vibrations of the $\mathrm{CF}_{2}$ group ascribed to the $\alpha$ PVDF polymorph [6,7].

Characteristic bands from the $\beta$-phase have been identified at 840,745 , 510 , and $445 \mathrm{~cm}^{-1}$. The band at $840 \mathrm{~cm}^{-1}$ is assigned to a mixed mode of $\mathrm{CH}_{2}$ rocking and $\mathrm{CF}_{2}$ asymmetric stretching vibration; this mode is parallel to the chain axis $[3,4,7]$. The band at $745 \mathrm{~cm}^{-1}$ is assigned to a rocking mode [4]. The $510-\mathrm{cm}^{-1}$ band is assigned to a $\mathrm{CF}_{2}$ bending mode; this mode is perpendicular to the chain axis and parallel to the polar $b$-axis $[3,4,7]$. The mode at $445 \mathrm{~cm}^{-1}$, also characteristic of the $\beta$-phase, is parallel to the $a$-axis [4].

Table 1. Characteristic Bands with Specific Vibrational Modes and Crystalline Phases

\begin{tabular}{|c|c|c|c|c|}
\hline $\begin{array}{l}\text { Experimental } \\
\text { Wavenumber, } \\
\mathrm{cm}^{-1}\end{array}$ & Group & Vibration & Comments & Ref. \\
\hline 3016 & $\mathrm{CH}_{2}$ & Symmetric stretching & & 5,9 \\
\hline 2978 & $\mathrm{CH}_{2}$ & Asymmetric stretching & & 5,9 \\
\hline 1453 & $\mathrm{CH}_{2}$ & $\begin{array}{l}\text { In-plane bending or } \\
\text { scissoring }\end{array}$ & & 8,9 \\
\hline 1335 & $\mathrm{CH}_{2}$ & $\begin{array}{l}\text { Out-of-plane bending } \\
\text { (wagging or twisting) }\end{array}$ & & 8 \\
\hline 840 & $\mathrm{CH}_{2}, \mathrm{CF}_{2}$ & $\begin{array}{l}\mathrm{CH}_{2} \text { rocking and } \mathrm{CF}_{2} \\
\text { asymmetric stretching }\end{array}$ & $\begin{array}{l}\beta \text {-Phase (out-of-phase } \\
\text { combination) }\end{array}$ & $3,4,7$ \\
\hline 763 & & $\begin{array}{l}\text { In-plane bending or } \\
\text { rocking }\end{array}$ & $\alpha$-Phase & 5 \\
\hline 745 & & $\begin{array}{l}\text { In-plane bending } \\
\text { or rocking }\end{array}$ & $\beta$-Phase & 4 \\
\hline 677 & & & $\begin{array}{l}\text { Presence of head-to-head } \\
\text { and tail-to-tail configu- } \\
\text { rations }\end{array}$ & 6 \\
\hline 615 & $\mathrm{CF}_{2}, \mathrm{CCC}$ & $\begin{array}{c}\mathrm{CF}_{2} \text { bending and } \mathrm{CCC} \\
\text { skeletal vibration }\end{array}$ & $\alpha$-Phase (out of phase) & 5,7 \\
\hline 600 & & & $\beta$-Phase & 4 \\
\hline 510 & $\mathrm{CF}_{2}$ & Bending & $\beta$-Phase & $3,4,7$ \\
\hline 490 & $\mathrm{CF}_{2}$ & Bending and wagging & $\begin{array}{c}\alpha \text {-Phase (in-phase } \\
\text { combination) }\end{array}$ & 6,7 \\
\hline 445 & & & $\beta$-Phase & 4 \\
\hline
\end{tabular}


Irregularities of head-to-tail addition, leading to defect structures, can occur during polymerization for several reasons [9]. In polymers of the vinylidene class, sequence isomerism may occur. The transmission band at $677 \mathrm{~cm}^{-1}$ points to the presence of head-to-head and tail-to-tail configurations [6]. Such defects are produced during the polymerization process and reduce the dipole moment of the all-trans conformation. This peak is observable in both polarization directions and is relatively unaffected by the deformation process. The transmittance values of this peak were taken as the reference for calculation of relative changes in the intensities of the other peaks as the ratio of transmittance is not dependent on the sample thickness [10]. This methodology has been used to monitor the relative amount of $\beta$ - and $\alpha$-crystalline phases and amorphous phase [11].

\section{CH Stretching Vibrations}

In many simple compounds, the $\mathrm{CH}_{2}$ group gives rise to two frequencies in the $2800-3100-\mathrm{cm}^{-1}$ zone. The asymmetric and symmetric stretching vibrations of the $\mathrm{CH}_{2}$ group in the virgin sample are located, respectively, at $3016 \mathrm{~cm}^{-1}\left(\mathrm{v}_{a}\right.$ $\left.\mathrm{CH}_{2}\right)$ and $2978 \mathrm{~cm}^{-1}\left(v_{s} \mathrm{CH}_{2}\right)$ (Fig. 3) [5,9]. Symmetric vibrations are generally weaker than asymmetric vibrations since the former lead to less of a change in dipole moment. These frequencies vary by only a few wavenumbers from compound to compound $[8,9]$.

\section{Effect of the Applied Stress}

Samples with different levels of deformations were prepared and studied by FTIR and DSC. Deformations of 30\%, 35\%, 37.5\%, 40\%, 42.5\%, 50\%, 60\%, $62.5 \%, 67.5 \%, 72.5 \%, 75 \%, 110 \%, 130 \%$, and $140 \%$ (break point) were achieved by interrupting the transverse experiment shown in Fig. 1 on reaching the desired values. Note that, at such strain levels, the materials are in the plastic region (Fig. 1), and therefore the deformations are highly irreversible on releasing the stress.

The samples were first analyzed by FTIR in both polarizations. The idea was to follow the variations of the absorbance and energy of the identified modes and spectral regions with the applied deformation.

An unexpected and drastic effect was observed between the virgin film and the film deformed with the lowest strain: The FTIR spectra of the deformed samples for a given polarization direction seemed to be identical to the spectra of the undeformed samples in the other polarization. That is, a polarization switching seemed to occur (Figs. 4 and 5). 

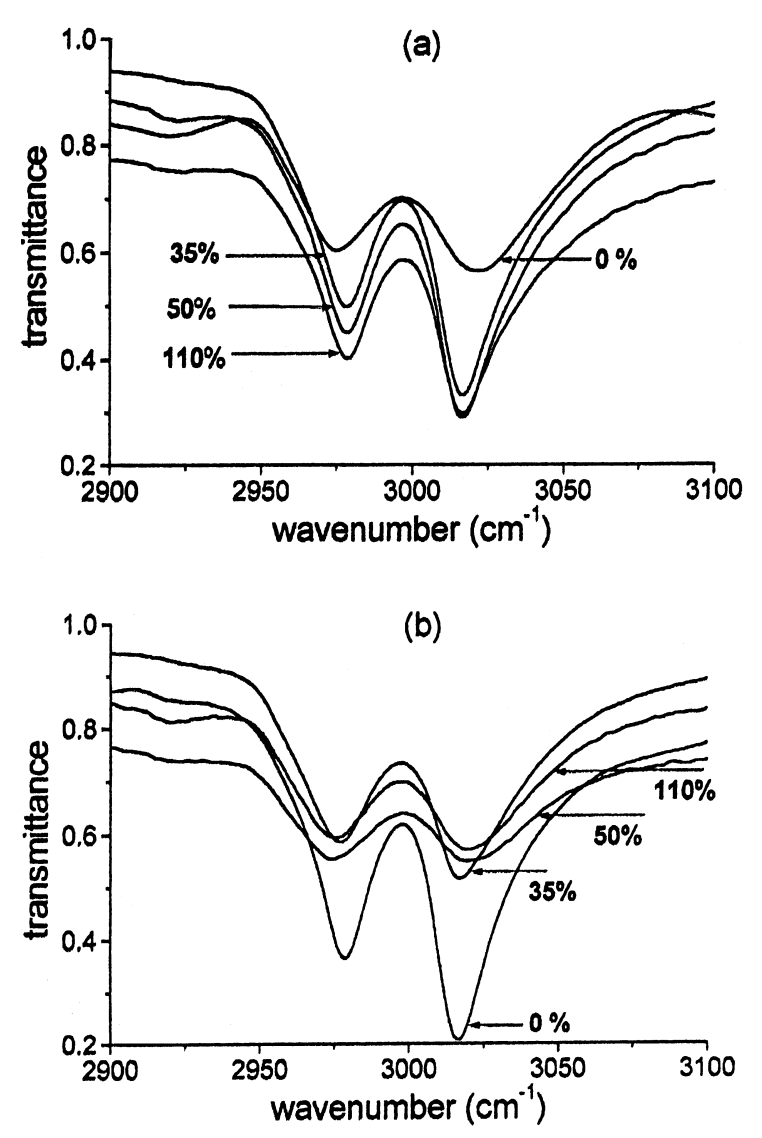

Figure 3. FTIR spectra of different samples of PVDF in the $\mathrm{C}-\mathrm{H}$ mode region. Comparison of three different deformations and virgin sample in both polarization directions: (a) the electric vector of the light is perpendicular to the chain orientation; (b) the electric vector of the light is parallel to the chain orientation.

Such inversion of behavior from longitudinal to transversal direction and vice-versa suggests changes of morphologies within the crystalline fraction such that the chains in the crystalline phase switch their direction by $90^{\circ}$. This inversion of the spectra occurs even for the lowest deformed sample $(\varepsilon=0.35)$, relative to the virgin one, (i.e., in the early plastic region). Moreover, the results in Figs. 4 and 5 show that, with increasing strain, the changes are less pronounced. Therefore, we may conclude that the inversion of polarization is not a progressive process, and it occurs possibly near the yield point of the specimens. A complete characterization of this process would need more morphological information than could be provided by small-angle X-ray scattering (SAXS) and wide-angle X-ray scattering (WAXS) experiments.

Many models have been proposed for the changes of microstructure during deformation. It is usually accepted that, in semicrystalline polymers, yielding is 


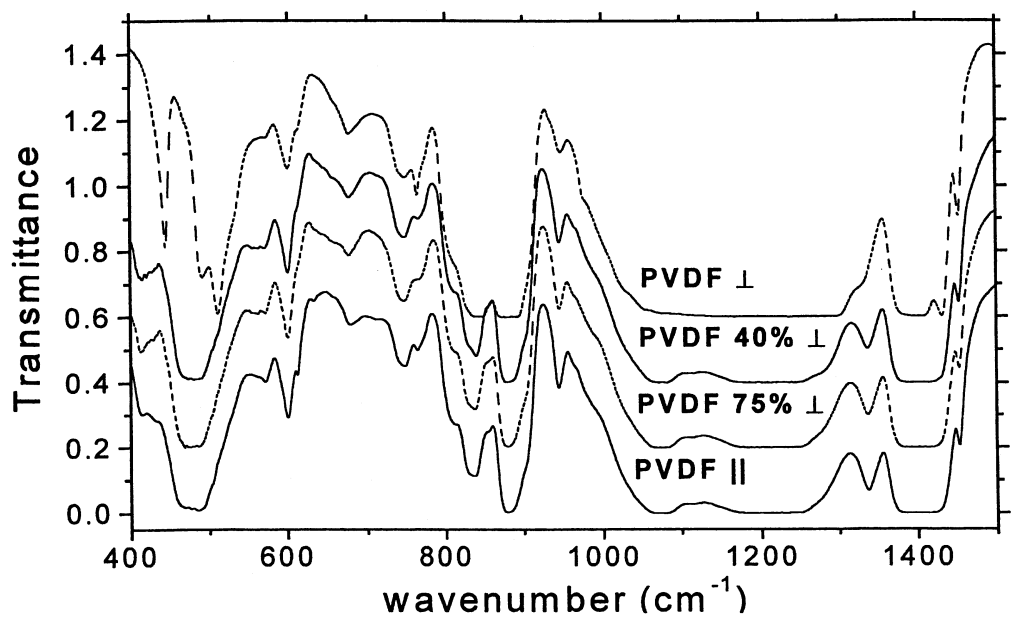

Figure 4. FTIR spectra of $\beta$-PVDF samples with different deformations. Comparison of two different deformations $(40 \%$ and $75 \%$ ) in perpendicular mode with a virgin sample in both polarizations.

associated with interchain sliding, chain segmental motions, and chain reconformation [12]. Slip can occur between the crystalline lamellae, which slide by each other, and within the individual lamellae by a process comparable to glide in monoatomic crystals. The last_-and dominant_-process leads to molecular orientation since the slip direction within the crystal is along the axis of the molecule. As plastic flow continues, the slip direction rotates systematically toward the tensile axis. The plastic deformation of semicrystalline polymers is a kinetic,

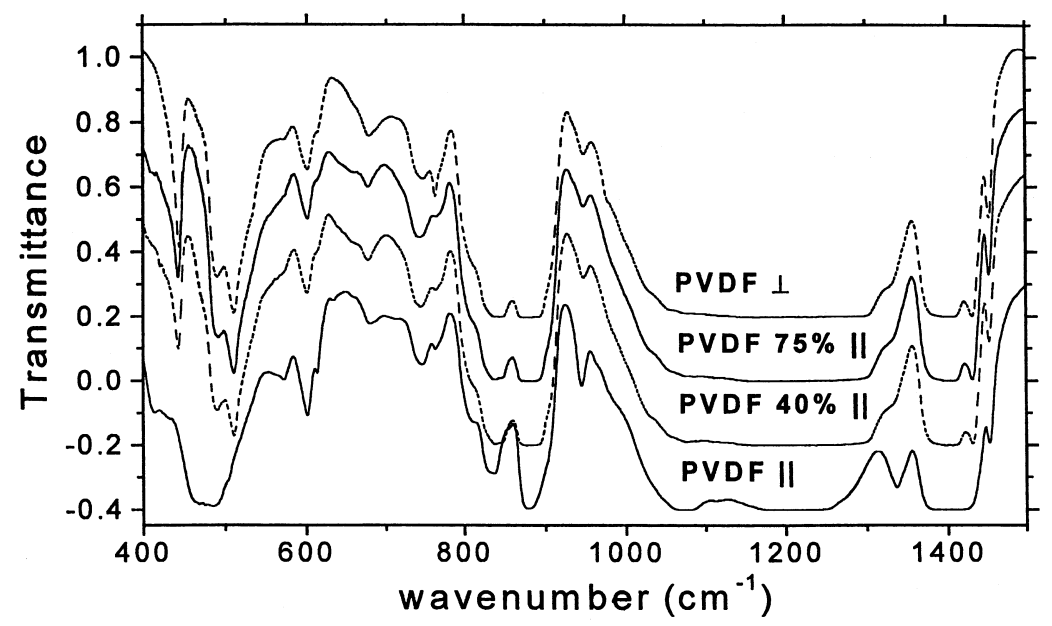

Figure 5. FTIR spectra of $\beta$-PVDF samples with different deformations. Comparison of two different deformations $(40 \%$ and $75 \%)$ in parallel mode with a virgin sample in both polarizations. 
thermoactivated process caused by shear stress [13]. Ultimately, the slip direction (i.e., the molecular axis) coincides with the tensile axis, and the polymer is then oriented and resists further extension.

The changes in the microstructure during deformation depend on the chemical nature of the material and on the initial morphology. In the case of the $\beta$ PVDF films studied in this work, the result suggests that yielding and further plastic drawing induce a complete reorientation of the crystal lamellae. There are models for deformation that are consistent with such features. For example, morphological changes in semicrystalline polymers during stress application have been studied by Flory and Yoon [14] and Takahashy and coworkers [15], who proposed that plastic deformation occurs by fusion and recrystallization of the crystal phase present in the semicrystalline polymer. This model was used to explain a local $90^{\circ}$ reorientation of the polymeric segments in the crystalline structure of PVDF [16], as seen by creep and stress-strain experiments.

The inversion effect observed in the FTIR experiments now provides a method for identifying the modes corresponding to the amorphous part of the polymer: These modes should not depend on the orientation of the sample or on the polarization switching that corresponds to a reorientation of the crystalline part of the polymer. The modes appearing, for example, at 600, 745, and 948 $\mathrm{cm}^{-1}$ are thus assigned to the amorphous component of the material.

\section{Differential Scanning Calorimetry Results}

DSC was used to characterize sections of previously deformed $\beta$-PVDF films. Only one endothermic peak was detected in the films (onset temperatures in the temperature range from $150^{\circ} \mathrm{C}$ to $160^{\circ} \mathrm{C}$ ), corresponding to the crystal melting. The DSC traces did not exhibit multiple melting endotherms that corresponded to different crystalline phases. Consequently, we can conclude that the $\beta$-phase was predominant in all the samples. Nevertheless, there was a very small amount of $\alpha$-phase present, as observed in the infrared spectra. The film orientation (deformation, in our case) changes the characteristic transition enthalpy, but not the melting temperature.

The degree of crystallinity was measured as the ratio between $\Delta H_{\mathrm{m}}$ and $\Delta H_{0}$, where $\Delta H_{\mathrm{m}}$ is the melting enthalpy of the material under study and $\Delta H_{0}$ is the melting enthalpy of totally crystalline material $\left(\Delta H_{0}=104.50 \mathrm{~J} / \mathrm{g}\right.$ for PVDF) $[17,18]$. It is to be noticed that the melting enthalpy that is reported is not explicitly assigned to the $\beta$-form of PVDF. In fact, to our knowledge, the true melting enthalpy for such material has never been measured.

As can be seen in Table 2, the melting enthalpy and consequently the degree of crystallinity of PVDF decrease with increasing applied deformation. This indicates that the deformation process leads to partial destruction of the crystalline structure. 
Table 2. Melting Temperature (Onset) and Enthalpy Measured by Differential Scanning Calorimetry for Samples Deformed up to Different Strains

\begin{tabular}{lccc}
\hline Strain, $\%$ & $T_{\mathrm{m}},{ }^{\circ} \mathrm{C}$ & $\Delta H_{\mathrm{m}}, \mathrm{J} / \mathrm{g}$ & Crystallinity, \% \\
\hline 0 & 157.9 & 50.0 & 47.9 \\
30 & 156.8 & 44.9 & 43.0 \\
60 & 157.7 & 43.5 & 41.6 \\
140 & 157.9 & 37.4 & 35.8 \\
\hline
\end{tabular}

However, the melting temperature was found to be constant for all samples, within the experimental error. It is known, for semicrystalline polymers in general and particularly for PVDF [19], that the melting temperature is related with the lamellae thickness. The results shown in Table 2 suggest that the strong changes in the morphology during the film drawing were not accompanied by changes in the crystalline lamellae thickness.

\section{CONCLUSIONS}

Films of semicrystalline PVDF in the $\beta$-phase were studied by FTIR and DSC to understand better the structural changes occurring during the deformation process. FTIR spectra showed a reorientation of the chains from perpendicular to parallel to the stress orientation within the plastic region. A decrease in the degree of crystallinity due to the deformation was observed. On the other hand, the thickness of the lamellae seemed to remain practically unchanged.

\section{ACKNOWLEDGMENT}

We thank José Luis Ribeiro and Luís Vieira for their help with the FTIR measurements. The work was partially supported by Fundação para a Ciência e Tecnologia (PRAXIS/P/CTM/14171/1998) and by a NSF grant (DMR-9520251).

\section{REFERENCES}

1. Nalwa, H.S. Recent Developments in Ferroelectric Polymers. J. Macromol. Sci. 1991, C31 (4), 341-432.

2. Vinogradov, A.; Holloway, F. Electro-mechanical Properties of the Piezoelectric Polymer PVDF. Ferroelectrics 1999, 226, 169-181.

3. Chi Chen, G.; Su, J.; Fina, L.J. FTIR-ATR Studies of Drawing and Poling in Polymer Bilaminate Films. J. Polym. Sci., Polym. Phys. 1994, 32, 2065-2075.

4. Bharti, V.; Kaura, T.; Nath, R. Ferroelectric Hysteresis in Simultaneously Stretched 
and Corona-Poled PVDF Films. IEEE Trans. Dielectrics Elec. Insulation 1997, 4 (6), 738-741.

5. Betz, N.; Le Moel, A.; Balanzat, E.; Ramillon, J.M.; Lamotte, J.; Gallas, J.P.; Jaskierowicz, G. A FTIR Study of PVDF Irradiated by Means of Swift Heavy Ions. J. Polym. Sci., Part B: Polym. Phys. 1994, 32, 1493-1502.

6. Hilczer, B.; Kulek, J. The Effect of Dielectric Heterogeneity on Pyroelectric Response of PVDF. IEEE Trans. Dielectrics Elec. Insulation 1998, 5 (1), 45-50.

7. Mattsson, B.; Ericson, H.; Torell L.M.; Sundholm, F., Micro-Raman Investigations of PVDF-Based Proton-Conducting Membranes. J. Polym. Sci., Part A: Polym. Chem. 1999, 37, 3317-3327.

8. Silverstein, R.M.; Webster, F.X. Spectrometric Identification of Organic Compounds; John Wiley and Sons: New York, 1998.

9. Garton, A. Infrared Spectroscopy of Polymer Blends, Composites and Surfaces; Hanser Editorial: Munich, 1992

10. Bower, D.I.; Maddams, W.F. Vibrational Spectroscopy of Polymers; Cambridge University Press: Cambridge, UK, 1989.

11. Lanceros-Méndez, S.; Mano, J.F. In preparation.

12. Liu, Y.; Truss, R.W. Study of Tensile Yielding of Isotactic Polypropylene. J. Polym. Sci., Polym. Phys. Ed. 1994, 32, 2037-2047.

13. Bershtein, V.A.M.; Egorov, V.M. Differential Scanning Calorimetry of Polymers; Ellis Horwood Series: Chichester, 1994.

14. Flory, P.J.; Yoon, D.Y. Nature 1978, 272, 226.

15. Takahashy, Y.; Zakoh, T.; Hanatani, N. Molecular Mechanisms for Elongation: PVDF Form II. Colloid Polym. Sci. 1991, 269, 781-784.

16. Hellinckx, S.; Bauwens, J.-C. The Yield Behaviour of PVDF and the Deformation Process at High Temperature. Colloid Polym. Sci. 1995, 273, 219-226.

17. Teyssedre, G.; Bernes, A.; Lacabanne, C. Influence of the Crystalline Phase on the Molecular Mobility of PVDF. J. Polym. Sci., Part B: Polym. Phys. 1993, 31, 2027-2034.

18. Nakagawa, K.; Ishida, Y. Dielectric Relaxation and Molecular Motions in Polyvinylidene Fluoride with Crystal Form II. J. Polym. Sci., Polym. Phys. Ed. 1973, 11, $1503-1533$.

19. Nakagawa, K.; Ishida, Y.J. Annealing Effects in Polyvinylidene Fluoride as Revealed by Specific Volume Measurements, Differential Scanning Calorimetry and Electron Microscopy. Polym. Sci., Polym. Phys. Ed. 1973, 11, 2153. 